\title{
A DOR E O RECÉM-NASCIDO DE RISCO: PERCEPÇÃO DOS PROFISSIONAIS DE ENFERMAGEM
}

Marly Veronez ${ }^{1}$, Darci Aparecida Martins Corrêa ${ }^{2}$

\begin{abstract}
RESUMO: Estudo descritivo, qualitativo com o objetivo de descrever a percepção dos profissionais de enfermagem que atuam em unidade de terapia intensiva neonatal sobre a dor no recém-nascido. Participaram 25 profissionais de um hospital universitário, os dados foram coletados nos meses de dezembro de 2008 e janeiro de 2009 por meio de questionário. Emergiram quatro categorias: nocicepção no período neonatal; percepção da dor nos recém-nascidos e suas consequências, procedimentos mais dolorosos realizados; e estratégias utilizadas para o manejo da dor. Os resultados envolvem o reconhecimento da dor pelos participantes, a utilização de medidas farmacológicas e não-farmacológicas, a punção venosa como procedimento mais doloroso e a necessidade de reconhecerem, avaliarem e intervirem neste fenômeno, considerando a implantação emergencial de um protocolo de avaliação da dor na unidade e treinamento da equipe envolvida, contribuindo para a humanização da assistência.
\end{abstract}

PALAVRAS-CHAVE: Dor; Recém-nascido; Unidades de terapia intensiva neonatal; Enfermagem; Humanização da assistência.

\section{PAIN AND THE NEWBORN AT RISK: NURSING PROFESSIONAL'S PERCEPTIONS}

\begin{abstract}
It's a qualitative study, which aim is to describe the newborn intensive care unit nursing professional's perception about the pain of the newborn. 25 professionals that work in a university hospital joined the research. Data were collected with the use of a questionnaire, during December 2008 and January 2009. Four categories emerged by the analysis: nociception at the neonatal period; pain perception of the newborn and its consequences; most painful procedures performed; strategies to manage pain. Results show the acknowledgement of the pain by participants, the use of pharmaceutical and non pharmaceutical measures, venipuncture as the most painful procedure, and the need for them to recognize, assess and intervene in this phenomena, considering the prompt implantation of a pain assessment guideline at the unit, as well as the training of the team, which will contribute to the humanization of care.
\end{abstract}

KEYWORDS: Pain; Newborn; Neonatal intensive care unit; Nursing; Humanization of care.

\section{EL DOLOR Y EL RECIÉN-NACIDO DE RIESGO: PERCEPCIÓN DE LOS PROFESIONALES DE ENFERMERÍA}

RESUMEN: Estudio descriptivo, cualitativo con el objetivo de describir la percepción de los profesionales de enfermería que actúan en unidad de terapia intensiva neonatal sobre el dolor del recién-nacido. Participaron 25 profesionales de un hospital universitario, los datos fueron recogidos en los meses de diciembre de 2008 y enero de 2009 por medio de cuestionario. Emergieron cuatro categorías: nocicepción en el periodo neonatal; percepción del dolor en los reciénnacidos y sus consecuencias, procedimientos más dolorosos realizados; y estrategias utilizadas para el manejo del dolor. Los resultados envuelven el reconocimiento del dolor por los participantes, la utilización de medidas farmacológicas y no farmacológicas, la punción venosa como procedimiento más doloroso y la necesidad de reconocer, evaluar e intervenir en este fenómeno, considerando la implantación de emergencia de un protocolo de evaluación del dolor en la unidad y entrenamiento del equipo envuelto, contribuyendo para la humanización de la atención.

PALABRAS CLAVE: Dolor; Recién-nacido; Unidades de terapia intensiva neonatal; Enfermería; Humanización de la atención.

\footnotetext{
${ }^{1}$ Enfermeira do Hospital Universitário de Maringá. Especialista em Enfermagem do Trabalho.

${ }^{2}$ Enfermeira. Doutora em Enfermagem. Professor Adjunto do Departamento de Enfermagem da Universidade Estadual de Maringá-UEM.
}

Autor correspondente:

Marly Veronez

Hospital Universitário de Maringá

Rua Pioneiro Darville Antonio Huergo, 1743 - 87083-210 - Maringá-PR, Brasil

Recebido: 26/10/09

Email:marlyveronez@gmail.com

Aprovado: 06/04/10 


\section{INTRODUÇÃO}

A dor não apresenta limite de idade, não tem preferência por sexo e é uma experiência de caráter universal, ocorrendo todas as vezes em que há uma agressão, seja ela, física, química, mecânica ou psíquica. Ainda hoje existem obstáculos para o efetivo tratamento da dor em pediatria, ou seja, mitos de que recém-nascidos (RN) e lactentes não sentem dor da mesma forma que os adultos ${ }^{(1-2)}$.

As vias anatômicas responsáveis pela dor (neurotransmissores, ramificações dentríticas e talâmicas) já se encontram desenvolvidas de forma precoce na $7^{\mathrm{a}}$ semana de gestação, e totalmente espalhadas pela superfície corporal ao redor da $20^{\mathrm{a}}$ semana de gestação e, portanto, os RN e lactentes podem sentir dor ${ }^{(2)}$.

A aplicação de estímulos nocivos no organismo provoca a ativação de receptores específicos, os nociceptores (terminações nervosas livres), que são encontrados ao longo dos tecidos do corpo. Os nociceptores transmitem informações através das fibras nervosas especializadas, chamadas de fibras sensoriais; fibras A-delta e fibras C. As fibras A-delta, que são mielinizadas e conduzem o impulso doloroso rapidamente, transmitem a dor aguda; as fibras C não são mielinizadas e conduzem o impulso mais lentamente, transmitindo as dores menos agudas, sensações de queimação e a dor crônica ${ }^{(3)}$.

Tradicionalmente, a falta de mielinização no RN tem sido proposta como um indicador de imaturidade do sistema nervoso central e para apoiar o argumento de que o RN não é capaz de sentir dor. É importante lembrar que os impulsos nociceptivos nos adultos também são conduzidos por fibras não mielinizadas e levemente mielinizadas. A velocidade de condução mais lenta nos nervos neonatais e nos feixes nervosos centrais, resultantes da mielinização incompleta, é compensada pelas distâncias interneurais mais curtas a serem percorridas pelo impulso nos $\mathrm{RN}^{(4)}$.

Os RN internados na Unidade de Terapia Intensiva Neonatal (UTIN) são expostos rotineiramente a inúmeros procedimentos e intervenções que causam estresse e dor ${ }^{(5)}$. Pesquisadores acreditam que estímulos dolorosos repetitivos influenciam na organização do cérebro e das fibras nervosas transmissoras desses estímulos $^{(6)}$. Estímulos dolorosos agudos desencadeiam nos RN uma resposta global ao estresse, que inclui modificação a nível cardiovascular, respiratório, imunológico, hormonal e comportamental, entre outros. Essas respostas fisiológicas são acompanhadas de uma re- ação endócrino-metabólica de estresse, com liberação, entre outros hormônios, de adrenalina, noradrenalina e cortisol, podendo também resultar em hiperglicemia e catabolismo protéico lipídico, interferindo no equilíbrio homeostático, que no RN já é precário(7).

Com base nestes fatores, surge a necessidade de uma atenção especial aos RN que se encontram hospitalizados em UTIN. A equipe de enfermagem atua nos cuidados diretos a estes bebês cabendo, portanto, a responsabilidade de estar atenta à presença de dor para intervir com medidas que possam colaborar com sua melhora clínica. Desta forma, é necessário manter o RN, o tanto quanto possível, estável do ponto de vista neurológico e comportamental, oferecendo assim um cuidado humanizado.

Atualmente, existem muitas alternativas disponíveis para evitar a dor e o sofrimento desnecessário dos neonatos hospitalizados, pois além da terapêutica medicamentosa, a equipe, principalmente a de enfermagem, pode valer-se de medidas alternativas e alívio efetivo do desconforto e da dor ${ }^{(8)}$.

Diante do exposto, este estudo teve por objetivo descrever a percepção dos profissionais de enfermagem que atuam na UTIN sobre a dor no RN.

\section{METODOLOGIA}

Trata-se de uma pesquisa descritiva com abordagem qualitativa. Este método favorece o aprofundamento do significado das crenças e dos valores dos indivíduos, permitindo o conhecimento do significado das ações e das relações humanas ${ }^{(9)}$.

Os critérios de inclusão dos profissionais da equipe de enfermagem foram atuar no cuidado direto ao RN na UTIN do Hospital Universitário Regional de Maringá (HURM), Estado do Paraná, concordar com e formalizar a participação no estudo. A equipe é formada por 13 enfermeiros assistenciais e 15 técnicos em enfermagem, distribuída em três turnos: manhã, tarde e noite.

As informações foram coletadas pela pesquisadora nos meses de dezembro de 2008 e janeiro de 2009, por meio de um questionário com questões semiestruturadas. A primeira parte abrangeu questões inerentes a aspectos pessoais, formação, tempo de formado e tempo de atuação na área de neonatologia. A segunda abordou o conhecimento e a percepção sobre a dor no RN, procedimentos mais dolorosos realizados, adoção ou não de instrumento para avaliar a dor, bem como estratégias utilizadas para o seu 
manejo. Os dados foram analisados qualitativamente, utilizando-se a técnica descritiva para a análise temática do conteúdo ${ }^{(10)}$. Após a leitura e releitura do material, os dados foram agrupados em categorias e analisados com base em referencial bibliográfico pertinente.

O projeto foi aprovado pelo Comitê de Ética em Pesquisa da Universidade Estadual de Maringá (Parecer n. 662/2008) e observaram-se os preceitos de ética em pesquisa. Todos os participantes, após terem sido orientados sobre o estudo, assinaram o Termo de Consentimento Livre e Esclarecido em duas vias, e na apresentação dos resultados estão identificados com nome de flores, por escolha da pesquisadora, e com anuência dos participantes, garantindo-lhes o anonimato.

\section{RESULTADOS E DISCUSSÃO}

Participaram do estudo 25 profissionais de enfermagem, três com menos de dois anos de experiência em neonatologia e os demais com 4 a 13 anos de experiência. Os resultados obtidos foram dispostos em quatro categorias, emersas das respostas dos informantes: Nocicepção no período neonatal; Percepção sobre a dor nos RN e suas consequências; Procedimentos mais dolorosos realizados nos RN; e Estratégias utilizadas para o manejo da dor.

\section{Nocicepção no período neonatal}

Todos os participantes acreditam que os RN sentem dor, porém, a maior parte respondeu ser essa dor igual à de adultos e neonatos. Este resultado merece atenção especial, uma vez que é sabido que o $\mathrm{RN}$, em especial o prematuro, sente mais dor que indivíduos de faixas etárias mais velhas, principalmente quando submetidos a procedimentos dolorosos ou desagradáveis, repetidamente ${ }^{(11)}$.

Isso ocorre devido à plena capacidade de percepção e à pouca capacidade de inibição da dor. No RN pré-termo extremo já existe a capacidade anatômica e funcional para a percepção dolorosa, com a produção de catecolaminas e de outras substâncias relacionadas ao estresse, podendo existir uma exarcebação da percepção dolorosa (hiperalgesia), devido a alterações químicas e estruturais, e que pode ser mantida por tempo prolongado. Muitas são as causas que contribuem para a dificuldade de inibição ou atenuação da sensação dolorosa: o sistema endorfínico não está completamente funcional; a imaturidade da inibição das aferências dolorosas descendentes corticais; a despolarização neuronal excessiva e o incompleto desenvolvimento do hipotálamo ${ }^{(12)}$.

Desta forma, a falta de inibição do estímulo nociceptivo aferente, pelas vias corticais descendentes, significa que não há atenuação do impulso doloroso. Neste sentido, mesmo que o RN exiba resposta clara e definida ao estímulo doloroso, essa resposta não é sempre previsível e organizada, e a falta de inibição dos impulsos sensoriais desencadeia respostas exageradas e generalizadas, especialmente no prematuro. Por isso, esses prematuros podem sentir mais dor que os pacientes mais velhos, uma vez que cada novo estímulo não é inibido e sim ampliado. Com a multiplicação de estímulos dolorosos, ou estímulos desagradáveis, e não intrinsecamente dolorosos, como a troca de fraldas ou a manipulação para pesar, podem passar a ser percebidos pelo bebê como dor ${ }^{(5)}$.

\section{Percepção sobre a dor nos recém-nascidos e suas consequências}

Cada profissional da equipe de enfermagem percebe a dor baseado em sua vivência profissional e científica, bem como pela influência cultural. Em suas manifestações, eles revelaram que a dor é percebida por eles mediante alterações comportamentais e fisiológicas dos RN. Dentre as alterações comportamentais destacaram o choro, expressão facial, resposta motora e irritabilidade.

[...] percebo a dor no bebê pelo choro [...] pela resposta motora [...]. (Violeta)

Quando está com dor [...] às vezes o choro é silencioso [...] com lágrimas. (Angélica)

Identifico a dor [...] pelo choro, irritabilidade. (Margarida)

O choro é considerado o método primário de comunicação do RN por mobilizar o adulto, seja a mãe ou o profissional cuidador. Neste sentido, um dos problemas que mais limitam o choro, como parâmetro para diagnosticar a dor, é o fato de que 50\% dos bebês não choram durante um procedimento doloroso; e ainda, o fato de que o choro pode ser desencadeado por outros estímulos não dolorosos, como a fome e o desconforto(5).

Estudo sobre a avaliação e alívio da dor em RN, realizado em um hospital da grande São Paulo, mostrou que $23,2 \%$ dos enfermeiros entrevistados citaram o 
choro e a expressão facial como parâmetros principais para avaliar a dor ${ }^{(13)}$. Tais resultados corroboram com 0 estudo em questão, como demonstram as falas a seguir:

Quando o bebê está sentido dor ele enruga a testinha, chora [...]. (Tulipa)

Identifico a dor pelas expressões da face, choro [...]. (Margarida)

[...] a expressão facial, pois ela é diferente dos bebês que choram sem dor. (Amor Perfeito)

O bebê apresenta faces de dor [...]. (Amarílis)

Para alguns entrevistados, além do choro e expressão facial, a irritabilidade e agitação foram lembradas como indicadores de dor ou alterações significativas a serem observadas nos RN:

[...] fica muito agitado, estressado [...]. (Amarílis)

Identifico a dor [...] pela irritabilidade do bebê. (Margarida)

[...] a testa fica franzida e ele fica muito inquieto [...]. (Azaleia)

A dor é percebida [...] pela irritabilidade, contrações musculares [...]. (Copo-de-leite)

Muitos profissionais perceberam a dor através das alterações fisiológicas, sendo as alterações dos sinais vitais as mais citados. A nocicepção no RN resulta em respostas fisiológicas, humorais e imunitárias imediatas que podem estar associadas com efeitos prejudiciais $^{(4)}$ : aumento da frequência cardíaca e da pressão arterial e variação de pressão intracraniana; e o sistema respiratório com elevação do consumo de oxigênio, queda de saturação de oxigênio e alteração entre ventilação e perfusão. Estes estímulos dolorosos também se manifestam de outras formas, como diminuição da motilidade gástrica e hipercoagulabilidade, entre outras ${ }^{(5)}$. Diante do estímulo doloroso agudo, observa-se que os RN respondem com modificações em seus parâmetros fisiológicos.

[...] alterações dos sinais vitais, diminuição da $\mathrm{SPO}_{2}$, taquicardia, taquipneia [...]. (Begônia)

Durante o procedimento doloroso [...] ocorre aumento da $\mathrm{FC}$, alteração da $\mathrm{SPO}_{2}$. (Lírio)

[...] modificação da FC e FR [...] queda da $\mathrm{SPO}_{2}$ [...] sudorese. (Orquídea)

Durante o estímulo doloroso [...] os sinais vitais ficam instáveis [...]. (Gérbera)

Os RN de muito baixo peso são limitados na sua capacidade de tolerar ambientes estressantes e superestimulantes, apresentando respostas tipicamente exageradas ou desorganizadas. Ao interagir com o ambiente, podem responder de maneira organizada ou desorganizada ${ }^{(14)}$. Os relatos a seguir demonstram que os profissionais de enfermagem observam o comportamento dos RN em seu cotidiano e sabem que a dor pode acarretar prejuízos à vida destes bebês:

[...] aumenta o nível de estresse, prejudicando a organização cerebral [...]. (Lírio)

A criança fica traumatizada [...] mais sensível à dor [...]. (Dama-da-noite)

[...] sono perturbado, o bebê mais agitado [...]. (Dália)

[...] desorganização cerebral [...] podendo ocasionar depressão, insegurança [...]. (Orquídea)

A exposição repetida à experiência dolorosa pode ter efeitos de longo prazo no sistema de dor e no desenvolvimento como, por exemplo, a redução do limiar de dor e hiperalgesia. A redução da dor aumenta a homeostase, ou seja, a organização interna, estrutural e funcional do organismo, contribuindo para a manutenção do equilíbrio e a estabilidade do RN, e são essenciais para o cuidado e suporte aos neonatos imaturos, a fim de sobreviverem à rotina estressante da UTIN ${ }^{(15)}$.

A dor acarreta importantes repercussões para o RN, das quais se enfatiza o desenvolvimento cerebral prejudicado, o que ameaça a sua estabilidade fisiológica e ocasiona reflexos negativos, tais como problemas comportamentais. Esses serão percebidos apenas na infância, assim como problemas psiquiátricos, tais como ansiedade, depressão e esquizofrenia ${ }^{(16)}$. O manuseio excessivo pode provocar hipoxemia, apneia, bradicardia e comportamento de estresse, além de perturbar o ritmo do sono ${ }^{(14)}$.

Para mim o estresse no RN causa hipoxia e hemor- 
ragia intracraniana [...]. (Rosa)

[...] quando eles estão aqui conosco [...] ficam traumatizados e assustados. (Violeta)

Hiperatividade, baixo limiar à dor, estresse [...]. (Begônia)

\section{[...] sono perturbado. (Dália)}

Os bebês, quando expostos à estimulação nociceptiva repetida e/ou persistente, passam também a exibir reações comportamentais diante de estímulos táteis não dolorosos e de estímulos desagradáveis, como o barulho. Ou seja, em pacientes submetidos a múltiplos estímulos dolorosos, os não dolorosos passam a ser percebidos como dor e os estímulos dolorosos passam a ser desencadeados em limiares cada vez mais baixos, com efeitos deletérios cumulativos ${ }^{(17)}$.

\section{Procedimentos mais dolorosos realizados nos recém-nascidos}

Muitos procedimentos dolorosos são realizados em RN admitidos em UTIN, principalmente em bebês prematuros. Os profissionais entrevistados acreditam e percebem que os procedimentos invasivos causam dor. Dos procedimentos citados, a punção venosa foi descrita com maior frequência, seguidas pela coleta de exames, glicemia capilar, retirada de fitas adesivas da pele, aspiração traqueal, manipulação excessiva, curativos, flebotomias, drenagem de tórax, pequenas cirurgias sem analgesia ou mesmo com analgesia, procedimentos médicos em geral, entre outros. As respostas a seguir indicam estes achados:

[...] a punção venosa periférica [...] Punção venosa $e$ arterial [...] punção arterial e a glicemia capilar constante [...]. (Lírio)

[...] pequenas cirurgias com analgesia insuficiente ou mesmo sem analgesia. [...] a punção venosa periférica [...] punção venosa e arterial [...] punção arterial e a glicemia capilar constante [...]. (Amorperfeito)

[...] realização do cateter central de inserção periférica. (Cravo)

[...] realização de curativos, procedimentos médicos. (Tulipa)

Descolar fitas adesivas e esparadrapo da pele sem uso do benjoim. (Orquídea)

\section{[...] aspiração traqueal é muito dolorosa. (Rosa)}

[...] a punção venosa periférica [...] punção venosa $e$ arterial [...] punção arterial e a glicemia capilar constante [...].(Orquídea)

[...] drenagem de tórax, colocação de cateter invasivo [...]. (Gérbera)

Um estudo realizado na França com 413 RN demonstrou que os bebês prematuros nas unidades de cuidados especiais sofriam, em média, 16 procedimentos dolorosos e estressantes e que alguns experimentaram cerca de 62 destes procedimentos diariamente ${ }^{(6)}$. No estudo em questão, os participantes citaram 16 procedimentos dolorosos diferentes que fazem parte da rotina diária.

Como se pode perceber, os procedimentos dolorosos são rotina em UTIN, assim, estratégias de prevenção da dor devem ocorrer, pois seu alívio, além de ser uma necessidade básica, é também um direito de todo ser humano.

\section{Estratégias utilizadas para o manejo da dor}

O resultado mostra que a maioria dos participantes utiliza-se de alguma estratégia para o manejo da dor nos RN. Contudo, alguns participantes responderam que não utilizam medidas preventivas na UTIN, o que demonstra não haver um protocolo a ser seguido para o alívio deste agravo, cada um avalia subjetivamente. Assim, os RN ficam à mercê de cada cuidador. Entre as estratégias utilizadas foram citadas as intervenções não-farmacológicas e as farmacológicas. As intervenções não farmacológicas ${ }^{(3)}$ têm como finalidade prevenir ou reduzir a intensidade de um processo doloroso, o que pode ser observado nas respostas da maioria:

[...] aplico medidas de conforto, carinho, mas observo que nem todos os profissionais envolvidos no cuidado fazem isso. (Dama-da-noite)

Acalento. Pego no colo após os procedimentos [...] eu envolvo o bebê no cueiro [...]. (Jasmim).[...] 
sucção não nutritiva, deixo o ambiente calmo $e$ tranquilo. (Amor-perfeito)

Ofereço conforto, converso com ele, toco como que para proteger [...]. (Violeta)

Procedimentos dolorosos ou estressantes devem ser minimizados e coordenados com outros aspectos da assistência ao RN. Uma possibilidade é agrupar a realização de intervenções dolorosas antes de um evento agradável como, por exemplo, alimentar ou segurar o bebê, envolvê-lo em fralda durante o procedimento e promover sucção não nutritiva. Após o procedimento, reduzir o barulho e a luz do ambiente, tocar, proporcionar contato mãe-bebê, pele a pele (método canguru), e levar ao colo ${ }^{(18)}$.

Neste sentido, é importante enfatizar que o tratamento da dor se inicia por ações e atitudes de humanização, pela redução do ruído e da luz, pela observação de protocolos de intervenção mínima do $\mathrm{RN}$, e pela abordagem não-farmacológica da dor que alcança a terapêutica analgésica ou anestésica. $\mathrm{O}$ carinho, assim como as medidas não-farmacológicas, devem fazer parte da rotina das UTIN, cabendo à enfermeira promovê-las por meio de capacitação da equipe e do fazer cotidiano ${ }^{(16)}$.

Outra intervenção referida foi o uso da glicose, embora na UTIN em estudo não exista um protocolo sobre o volume e dose a serem ofertados; esta solução não-farmacológica é usada como medida preventiva primordial para o alívio da dor; mas seu uso é reconhecido e recomendado pelas Sociedades Americanas e Canadenses de Pediatria ${ }^{(19)}$. Eis alguns procedimentos relatados na pesquisa:

[...] pingar duas gotinhas de glicose na boquinha antes de puncionar. (Violeta)

[...] uma gotinha de glicose via oral durante os procedimentos. (Dama-da-noite)

Ofereço gotinhas de glicose 25\%; sucção não nutritiva [...]. (Orquídea)

Administro glicose 50\% via oral [...] acalento [...]. (Jasmim)

O estudo demonstrou que o açúcar favorece a liberação de endorfinas, substâncias analgésicas produzidas pelo próprio corpo, e que geram sensação de bem-estar. O uso da solução de sacarose a $25 \%$ $(0,5 \mathrm{ml} / \mathrm{kg})$ mostrou-se eficaz, quando administrada por via oral dois minutos antes do procedimento invasivo; nestes casos, observou-se que a frequência cardíaca variou menos e não houve quase queda nos níveis de saturação de oxigênio. Enfatiza o estudo que não é possível eliminar procedimentos invasivos e dolorosos, porque são extremamente necessários para os bebês de risco mas, de certa maneira, se pode prevenir alguns efeitos negativos, protegendo desta forma o bebê $\hat{e}^{(20)}$.

Em relação ao uso de medidas farmacológicas, os profissionais revelaram que solicitam aos médicos a prescrição de agentes farmacológicos e/ou administram os que já estão prescritos. Dores intensas devem ser manejadas com agentes farmacológicos, enquanto as dores menores podem ser manejadas por meio de medidas não-farmacológicas de alívio e prevenção ${ }^{(15)}$ :

Solicito para o médico prescrever analgésico [...]. (Jasmim)

[...] administro medicamentos para dor. (Lágrimasde-Cristo)

Eu solicito uso de medicações específicas para dor [...]. (Orquídea)

[...] quando não está prescrito analgésico solicito ao médico [...]. (Margarida)

O tratamento farmacológico inclui o uso de analgésicos não opioides que atuam por meio da inibição das prostaglandinas e do tromboxane, liberados durante a agressão tecidual; eles estão indicados em processos dolorosos leves ou moderados ou quando a dor está associada a um processo inflamatório. Os analgésicos opioides atuam nos receptores especificamente ligados à analgesia; sua interação com outros tipos de receptores opioides desencadeia, de maneira paralela à analgesia, depressão respiratória, retenção urinária, tolerância e dependência física. Por isso, é necessário conhecer bem os mecanismos de ação dos opioides para que se possa utilizá-los de maneira segura ${ }^{(5)}$. Porém, não há indicações absolutas para o emprego de analgésicos em RN; a decisão a respeito de aliviar a dor deve ser individualizada, mas não esquecida ${ }^{(12)}$.

\section{CONSIDERAÇÕES FINAIS}

O estudo demonstrou que todos os participantes 
reconhecem que os RN são capazes de sentir dor, porém houve discordância quanto a considerar que a dor é igual em adultos e crianças. Os RN, quando internados, demonstram a dor através de alterações comportamentais e fisiológicas, sendo estas percebidas pela maioria dos profissionais.

Identificamos vulnerabilidade no conhecimento dos profissionais de enfermagem que atuam na UTIN no que se refere à dor e analgesia do RN, essencial à compreensão da necessidade de identificar e tratar esse fenômeno tão complexo, embora identifiquem e utilizem estratégias para o seu controle.

Talvez isso esteja relacionado à deficiência do estudo desta temática durante a formação profissional, dificultando, portanto, que medidas de intervenções ocorram para o manejo da dor. A inexistência de protocolos de avaliação e tratamento da dor corrobora para a limitação de sua identificação e manejo.

Para finalizar, acreditamos que os objetivos do trabalho foram atingidos e que os aspectos assinalados possam despertar os profissionais de enfermagem a reconhecer, avaliar e intervir neste fenômeno, e que sem a adoção rotineira de escalas de avaliação da dor na UTIN, a dor do RN ficará dependendo sempre da observação de cada cuidador responsável. Assim, devemos considerar a implantação emergencial destas escalas nas unidades, e promover treinamento da equipe envolvida nestes cuidados para uniformizar as medidas adotadas.

\section{REFERÊNCIAS}

1. Barbosa SMM. Dor em pediatria. In: Neto OA, Costa CMC, José TTS, Siqueira JTT, colaboradores. Dor: princípios e prática. Porto Alegre: Artmed; 2009. p.77983.

2. Brock R. Dor. In: Basseto MCA, Brock R, Wajnsztejn R. Neonatologia: um convite à atuação fonoaudiológica. São Paulo: Lovise; 1998. p.201-4.

3. Tamez RN, Silva MP. Enfermagem na unidade de terapia intensiva neonatal: assistência ao recém-nascido de alto risco. $2^{\mathrm{a}}$ ed. Rio de Janeiro: Guanabara Koogan; 2002. p.45-51.

4. Colhado OCG. Dor no recém-nascido: fisiopatologia, avaliação e tratamento. Rev Dor. 2004; 5(2):286-94.

5. Guinsburg R. Dor no recém-nascido. PRORN. Programa de atualização em neonatologia. Porto Alegre. Ciclo 3. Módulo 3; 2006. p.9-45.
6. Carbajal R, Rousset A, Danan C, Coquery S, Nolent P, Ducrocq S, et al. Epidemiology and treatment of painful procedures in neonates in intensive care units. JAMA. 2008;300(1):60-70.

7. Guinsburg R. Dor no recém-nascido: importância do estudo da dor no recém-nascido. In: Rugolo LMS. Manual de neonatologia. Sociedade de Pediatria de São Paulo. Departamento de Pediatria. Rio de Janeiro: Revinter; 2000. p.63-9.

8. Gaiva MAM. O cuidar em unidades de cuidados intensivos neonatais: em busca de um cuidado ético e humanizado. Cogitare Enfem. 2006;11(1):61-6.

9. Minayo MCS. O desafio do conhecimento: pesquisa qualitativa em saúde. 9a ed. São Paulo: Hucitec; 2006.

10. Bardin L. Análise de conteúdo. Lisboa: Edições 70; 2004.

11. Guinsburg R, Balda RCX. Dor em neonatologia. In: Teixeira MJ. Dor. Curitiba: Maio; 2003. p. 547-53.

12. Ministério da Saúde (BR). Secretaria de Políticas de Saúde. Área de saúde da criança. Atenção humanizada ao recém-nascido de baixo peso: método mãe canguru: manual do curso/ Secretaria de Políticas de Saúde, área da Saúde da Criança. Brasília: Ministério da Saúde;2002. p.105-37.

13. Crescêncio EP, Zanelato S, Leventhal LC. Avaliação e alívio da dor no recém-nascido. Rev Eletron Enferm. [Internet]. 2009;11(1) [acesso em 10 mar 2009]. Disponível: http://www.fen.ufg.br/revista/v11/n1/pdf/ v1n1a08.pdf

14. Daré Júnior S, Imamura PEA, Figueira BBD, Fernandes GCV. Humanização e abordagem desenvolvimentista nos cuidados ao recém-nascido de muito baixo peso. In: Costa, HPF, Costa STM, coordenadores. O recémnascido de muito baixo peso. São Paulo: Atheneu; 2004. p.85-95.

15. Gaspardo CM, Linhares MBM, Martinez FE. A eficácia da sacarose no alívio de dor em neonatos: revisão sistemática da literatura. J Pediatr. 2005;81(6):435-42.

16. Sousa BB, Santos MH, Sousa FGM, Gonçalves APF, Paiva SS. Avaliação da dor como instrumento para cuidar de recém-nascidos pré-termo. Texto Contexto Enferm. 2006;15 (n.esp):88-96.

17. Guinsburg R; Leslie ATS, Covolan L. Consequências da dor repetida ou persistente no período neonatal. Rev Eletr Jorn Cient [Internet] 2007 [acesso em 19 fev 
2009]. Disponível: http://www.comciencia.br/comciencia/ ?section=8\&edicao=24\&id=269.

18. Marter LJV, Pryor CC. Tratamento da dor e do estresse na UTIN. In: Cloherty JP, Eichenwald EC, Stark AR. Manual de neonatologia. Rio de Janeiro: Guanabara Koogan; 2005. p.603-14.

19. American Academy of Pediatrics and Canadian Pediatric Society. Prevention and management of pain and stress in the neonate. Pediatrics. 2000;105:454-61.

20. Gaspardo CM, Miyase CI, Chimello JT, Martinez FE, Linhares MBM. Is pain relief equally efficacious and free of side effects with repeated doses of oral sucrose in preterm neonatos? Pain. 2008;137:16-25. 